\title{
Estrategias docentes de profesores universitarios en tiempos de Covid-19
}

\author{
Amawtakunap alliplulaykuna hatunyaćhaywasip \\ yaćhachikunap Covid - 19 pachankunaććhu \\ Kantagansipage irasi ogomeantaigatsi \\ kara ogomenotsipongopagekë kara pinanë \\ mantsigatagansijenga Kobi-19
}

Recepción: 12 diciembre 2020 Corregido: 16 enero 2021 Aprobación: 29 marzo 2021

Oscar Cencia Crispín

Nacionalidad: Peruana / Universidad Nacional del Centro del Perú Correo: ocencia@uncp.edu.pe / ORCID: https://orcid.org/oooo-0o02-0815-5934

Miriam Milagros Carreño Colchado

Nacionalidad: Peruana / Universidad Nacional Mayor de San Marcos Correo: mcarrenoc@unmsm.edu.pe / ORCID: https://orcid.org/oooo-ooo2-6261-3318

Pedro Eche Querevalú

Nacionalidad: Peruana / Universidad César Vallejo

Correo: peechequ@ucvvirtual.edu.pe. / ORCID: https://orcid.org/oooo-0003-4547-3998

Gustavo Isaac Barrantes Morales

Nacionalidad: Peruana / Universidad Privada del Norte

Correo: gustavo.barrantes@upn.edu.pe / ORCID: https://orcid.org/oooo-0002-3180-0604

Gardenia Giovana Cárdenas Baldeón

Nacionalidad: Peruana / Universidad Nacional Mayor de San Marcos Correo: gardenia.cardenas@unmsm.edu.pe / ORCID: https://orcid.org/oooo-0002-8862-1869

\section{Resumen}

El presente artículo de revisión trata acerca de las estrategias docentes en profesores universitarios en el contexto actual de la pandemia de la COVID-19, en el cual los docentes han asumido un rol importante en la continuidad pedagógica, desde el uso de estrategias (entendida como didáctica de la enseñanza), hasta los desafíos de adaptación a partir de la experiencia de una educación a distancia o no presencial, expresadas en actividades de aprendizaje de forma sincrónica y asincrónica. El objetivo de este artículo es describir el uso de las herramientas digitales que predominan en la práctica pedagógica en los profesores universitarios en tiempos de la COVID-19, sobre la base del estado del arte relacionado a la temática. La revisión de la literatura se desarrolló a través de la ficha de análisis de contenido, que tuvo como finalidad organizar información relevante respecto a las estrategias docentes que vienen usando los profesores al momento de desarrollar sus clases de manera virtual, a distancia o remoto, y donde se describe que el docente se reinventó para la nueva realidad pedagógica en favor de miles de estudiantes universitarios. Por último, se concluyó que no todos los estudiantes, al igual que los docentes, tienen las mismas condiciones tecnológicas y las competencias, capacidades, habilidades y destrezas digitales pertinentes para desarrollar sus clases de manera no presencial.

\section{Palabras clave:}

Estrategias docentes, profesores universitarios, educación y Covid-19.

\section{Lisichiku limaykuna:}

amawtakunap alliplulanin, hatunyaćhaywasip yaćhachininkuna, yaćhana Covid-19wan.

\section{Nibarintsi katingaro:}

Kanagantsipage

ogomeantaigatsi

ogomentosipongokë aike ogomeantagansi, kobi-19. 


\title{
Teaching Strategies of University Teachers in Times of Covid-19
}

\begin{abstract}
This review article deals with teaching strategies in university professors in the current context of the COVID-19 pandemic, in which teachers have assumed an important role in pedagogical continuity, from the use of strategies (understood as teaching didactics), to the challenges of adaptation from the experience of distance or non-face-toface education, expressed in synchronous and asynchronous learning activities. The objective of this article is to describe the use of digital tools that predominate in pedagogical practice at university professors in times of COVID-19, based on the state of the art related to the subject. The literature review was developed through the content analysis form, which was intended to organize relevant information regarding the teaching strategies that teachers have been using when developing their classes virtually, distance or remotely, and where it is described that the teacher reinvented himself for the new pedagogical reality in favor of thousands of university students. Finally, it was concluded that not all students, like teachers, have the same technological conditions and the relevant digital competencies, capacities, abilities and skills to develop their classes in a non-face-to-face way.
\end{abstract}

\section{Estratégias docentes de professores universitários em tempos de Covid-19}

\section{Resumo}

O presente artigo de revisão trata das estratégias docentes em professores universitários no contexto atual da pandemia COVID-19, em que os professores têm assumido um papel importante na continuidade pedagógica, desde o uso de estratégias (entendidas como didática do ensino), até os desafios da adaptação a partir da experiência de uma educação a distância ou não presencial, expressa em atividades de aprendizagem síncrona e assíncrona. O objetivo deste artigo é descrever o uso das ferramentas digitais predominantes na prática pedagógica de professores universitários no tempo do COVID-19, com base no estado da arte relacionado à temática. A revisão bibliográfica foi desenvolvida através do formulário da ficha de análise de conteúdo, que teve como finalidade organizar informações relevantes sobre as estratégias docentes que os professores vêm utilizando no momento do desenvolvimento de suas aulas de forma virtual, à distância ou remota, e onde é descrito que o professor se reinventou para a nova realidade pedagógica a favor de milhares de estudantes universitários. Por último, se conclui que, nem todos os estudantes, assim como os professores, possuem as mesmas condições tecnológicas e as competências, capacidades, habilidades e destrezas digitais pertinentes para desenvolver suas aulas de forma não presencial.
Keywords

Teaching

strategies, university professors, education and Covid-19.

\section{Datos de los autores}

Oscar Cencia Crispín es docente e investigador en educación. Doctor en Ciencias de la Educación, de la Universidad Nacional del Centro del Perú. Magister en Educación, mención Tecnología Educativa por la Universidad Nacional del Centro del Perú.

Miriam Milagros Carreño colchado es docente e investigadora en Periodismo y Comunicación. Doctoranda en Educación y Docencia Universitaria por la Universidad Nacional Mayor de San Marcos. Maestra en Periodismo y Comunicación Multimedia por la Universidad San Martín de Porres. Es Licenciada por la Universidad Jaime Bausate y Meza.

Pedro Eche Querevalú es docente e investigador en educación, tecnologías de la información y comunicación, proyectos educativos, pueblos originarios. Doctorando en Educación y Docencia Universitaria por la Universidad Nacional Mayor de San Marcos. Magister en Educación Tecnológica por la Universidad Nacional de Educación Enrique Guzmán y Valle, Ciudad de Lima. Perú.

Gustavo Isaac Barrantes Morales es docente e investigador en Comercio Exterior, entornos de aprendizaje y emprendimiento innovador. Doctorando en Educación y Docencia Universitaria por la Universidad Nacional Mayor de San Marcos. Magister en Gerencia de Operaciones y Logística. Licenciado en Administración de Negocios Internacionales por la Universidad de San Martín de Porres.

Gardenia Giovana Cárdenas Baldeón es investigadora en Ciencias Sociales. Doctoranda en Derecho y Ciencias Políticas por la Universidad Nacional Mayor de San Marcos. Maestra en Derecho Penal por la Universidad Nacional del Centro del Perú. Egresada de la maestría de Antropología Jurídica por la Universidad Nacional del Centro del Perú. 


\section{Introducción}

La práctica pedagógica de los docentes universitarios se ha adaptado al contexto actual, realidad que experimentan todas las sociedades en su proceso educativo. Es así que ahora la educación es esencialmente a distancia y, de acuerdo a la SUNEDU, "es la modalidad de estudios, basada en entornos virtuales de aprendizaje, que se caracteriza por la interacción síncrona (simultánea) y asíncrona (diferida), entre los docentes, estudiantes y materiales de enseñanza".

En el mundo contemporáneo, la pandemia de la COVID-19 ha generado cambios de paradigmas en todas las actividades del ser humano, en especial en el ámbito de la educación.

Desde la percepción de Pinheiro (2020)

los docentes tuvieron que reinventar e insertar nuevas formas de enseñar en su proceso de trabajo; tuvieron que discutir diferentes enfoques educativos $y$, dado las necesidades de reajustar los métodos de enseñanza en salud, insertaron tecnologías remotas como herramientas imprescindibles para satisfacer la necesidad real de continuidad de clases en modelo no presencial [uso de herramientas digitales]. Para muchos, es un desafío, ya que actualmente impregna una reflexión sobre la atención de formación a distancia. (p.141)

En consecuencia, existe la necesidad de comprender la interacción del docente con los estudiantes en esta época de la educación a distancia y virtual, caracterizada por el uso de plataformas de convergencia digital, interactivas, síncronas y asíncronas. Por ello,

la formación del docente debe ir pareja a los cambios didácticos y socioeducativos que se van generando en cada momento. De ahí, la importancia de la formación permanente del profesorado en todas las etapas educativas desde los principios de interacción, asimilación y acomodación (Akerlind, 2003; Coffey y Gibbs, 2000, citado por Vásquez, 2015, p.150).

Por lo tanto, el docente universitario debe tener en cuenta el dominio actualizado y científico en su área o especialidad; además de una formación actualizada para el uso de estrategias docentes, donde el profesor, los estudiantes y lo recursos didácticos, tengan conectividad; buscando que la institución educativa y el sistema educativo en general garantice todo el componente tecnológico para el uso, apropiación y desarrollo de la herramientas digitales, apoyados en las Tecnologías de la Información y Comunicación (TIC), cuyo escenario del proceso de enseñanza-aprendizaje, es una sala en la nube de internet o ciberespacio, propio de la educación virtual.

Una experiencia importante es la de la universidad alemana Universidad Tecnológica de Chemnitz, en donde se ha encontrado que:

el aprendizaje mejorado por la tecnología ya desempeñaba un papel importante en la mayoría de las universidades alemanas, la pandemia de la COVID-19 ha requerido expandir la infraestructura existente y resaltar áreas en las que las universidades deben enfocarse en su estrategia de digitalización. Además, este desafío ha acelerado enormemente la digitalización del sector de educación superior alemán. (Skulmowski y Daniel, 2020, p.212)

El problema formulado para el presente trabajo es ¿Cuáles son las estrategias docentes en profesores universitarios en tiempos de Covid-19?, teniendo como objetivo de este artículo describir el uso de las herramientas digitales que predominan en la práctica pedagógica en los profesores universitarios en tiempos de la COVID-19. 


\section{Metodología}

Pese a que existe investigación limitada sobre las estrategias docentes en profesores universitarios en el campo de la interacción activa en la educación a distancia y virtual en tiempos de la COVID-19, la presente investigación se ha trabajado desde la revisión y selección sistemática de artículos científicos referentes a este tema, que se han encontrado en revistas indexadas en la base de datos de Scopus, Scielo, Eric, Google Académico y en algunos repositorios de la UNMSM y la UNAM. Se ordenaron las fuentes y documentos confiables, siguiendo los criterios planteados por Garcés y Duque (2007, p.184), quiénes remarcan que es fundamental para la evaluación y el análisis crítico de la información "la calidad o nivel académico, originalidad, aporte al conocimiento, claridad en la presentación y redacción de ideas, pertinencia y dominio de bibliografía y el interés y actualidad del tema". Es decir, se trata de un diseño de análisis documental definido por Solís (2003) citado por Peña y Pirela (2007) como "la operación que consiste en seleccionar ideas informativamente relevantes de un documento a fin de expresar su contenido sin ambigüedades para recuperar la información contenida en él” (p. 59).

\section{Proceso de selección}

Para la obtención de la literatura seleccionada se realizó la búsqueda en las bases de datos mencionadas a través de las palabras claves: estrategias docentes, profesores universitarios, educación y Covid-19. Posteriormente se incluyó el operador booleano OR para enlazar las palabras claves y excluirse entre sí. Así mismo se descartó literatura científica que no tenían relación con la variable de estudio ni tampoco pertenecer al ámbito de educación superior a analizar.

\section{Resultados}

\section{Desarrollo de las clases en la universidad en un mundo digitalizado}

En la actualidad existe diversidad de herramientas tecnológicas que identifican la era contemporánea digital que viene transformando las actividades del hombre, en especial la forma en que los docentes y los estudiantes interactúan en el proceso de enseñanza-aprendizaje, a través de las denominadas tecnologías digitales creativas en la educación a distancia. En esta realidad de la COVID-19, el docente universitario necesita desarrollar competencias digitales para concretar su práctica pedagógica. En el documento Measuring Digital Skills across the EU: EU wide indicators of Digital Competence (European Commission, 2014), señalado por (López, Couso y Simarro, s/f) se consideran los siguientes indicadores para definir las competencias digitales:

- Información (Identificar, localizar, recuperar, almacenar, organizar y analizar información digital, juzgando su relevancia y propósito);

- comunicación digital (comunicarse en entornos digitales, compartir recursos a través de herramientas en línea, vincularse con otros y colaborar a través de herramientas digitales, interactuar y participar en comunidades y redes con sensibilización intercultural);

- creación de contenidos digitales (en múltiples formatos, integrando y reelaborando conocimientos y contenidos previos y teniendo en cuenta los derechos de propiedad intelectual y las licencias); 
- seguridad digital (protección personal, protección de datos, protección de identidad digital, medidas de seguridad, uso seguro y sostenible de las herramientas digitales);

- y resolución digital de problemas (identificar necesidades y recursos digitales, tomar decisiones informadas sobre cuáles son las herramientas digitales más apropiadas de acuerdo con el propósito o necesidad, resolver problemas conceptuales a través de medios digitales, usar creativamente tecnologías y resolver problemas técnicos).

\section{Suárez y otros (2019) refieren,}

sin embargo, con frecuencia se puede identificar que los docentes consideran tener competencias digitales cuando solo hacen uso de la tecnología para poner a sus estudiantes a realizar búsquedas o investigaciones en internet. Evidentemente el concepto es más amplio y complejo, tal como lo manifiesta Ferrari (2012): los discursos sobre la alfabetización tienden a centrarse en el argumento de la decodicación y codicación, es decir, en la lectura y escritura, pero aquí preferimos referirnos a la competencia digital como el conjunto de conocimientos, habilidades y actitudes necesarias hoy en día para ser funcional en un entorno digital. Por tanto, además de los cambios que introducen las nuevas tecnologías en la alfabetización de la lectura y escritura, podemos argumentar que la competencia digital requiere un conjunto nuevo de habilidades, conocimientos y actitudes (p. 16).

El desarrollo de estas nuevas tareas profesionales implica que el profesorado debe adquirir nuevas habilidades y competencias vinculadas con el saber utilizar pedagógicamente las tecnologías digitales (Area-Moreira, 2019, p. 17).

\section{Ventajas de la educación a distancia o no presencial:}

Como consecuencia de la COVID-19, y de las continuas restricciones de la asistencia de forma presencial a las escuelas y universidades, la educación en línea se ha convertido en la única opción para el desarrollo de las clases en las universidades y demás instituciones educativas. Esta educación en línea es un aprendizaje apoyado electrónicamente, que se basa en Internet para la interacción profesor / alumno y la distribución de materiales de clase más allá del campus universitario. Es importante mencionar que el sector educativo a nivel mundial fue uno de los sectores en asumir tardíamente las tecnologías digitales y que sólo el $3 \%$ de todo el presupuesto educativo a nivel del mundo lo destinaban a tecnologías digitales. Estás condiciones no favorecen el desarrollo de la enseñanza-aprendizaje en línea, generándose limitaciones, al acceso de dispositivos digitales y conexión a internet de alta velocidad, especialmente en las familias o países más pobres del mundo, además en esas condiciones el aprendizaje en línea era inferior a la enseñanza eminentemente tradicional. (Stub Sara, 2020, citado por Abreu, 2020, p. 13).

Una ventaja importante del desarrollo de la educación a distancia es el aprendizaje multimodal. Schnotz (2002) menciona que "las combinaciones de textos visuales y espaciales y otras formas de presentaciones visuales pueden apoyar la comunicación, el pensamiento y el aprendizaje sólo si existe una apropiada interacción con el sistema cognitivo del individuo" (p. 113).

Además, según lo manifestado por Copertari y Sgreccia (2011), la modalidad no presencial permite: 
un estilo de gestión académica al servicio de los estudiantes, aprendizaje colaborativo en red a través de los recursos pedagógicos y didácticos disponibles, el desarrollo de habilidades cognitivas y metacognitivas en los estudiantes que les ayudan a alcanzar una comprensión genuina orientada mediante acciones tutoriales, un sistema de evaluación procesual y permanente igual al de modalidades presenciales y flexibilización de la accesibilidad, permanencia y egreso de los estudiantes y recursos tecnológicos y didácticos que promueven la interactividad.

Por otro lado, Varguillas y Bravo (2020), señalan que las bondades de la modalidad de aprendizaje virtual son:

la abundancia de información web disponible en diferentes formatos, el uso de recursos tecnológicos adicionales a los de una clase presencial, como foros, chats y videoconferencias, la posibilidad de un aprendizaje más interactivo y participativo, desarrollo de la autonomía estudiantil y la retroalimentación asincrónica para aprovechar mejor el tiempo de los estudiantes.

Otras ventajas que deben señalarse dentro de la educación universitaria no presencial y virtual son: los docentes y los estudiantes están en la capacidad de interactuar desde la ubicuidad; existe mayor posibilidad de realizar trabajo colaborativo entre estudiantes; la flexibilidad al momento de desarrollar las clases; la personalización del aprendizaje y de los contenidos; aprendizaje multimodal y una comunicación en la diversidad de forma síncrona o asíncrona. Para que todo esto se realice, el docente deberá integrarse a la cultura digital mejorando su actitud hacia los métodos y los contenidos, desarrollando competencias digitales, reconfigurando la motivación y observación, y esencialmente adoptando estrategias híbridas en el proceso pedagógico.

En este sentido, la implementación de estrategias metodológicas como el aula invertida contribuye a que el estudiante se convierta en el protagonista de su propio aprendizaje, contando en su plataforma virtual con los elementos didácticos colocados anticipadamente por el profesor, siendo este último un guía y curador de contenidos y experimentando en las clases sincrónicas actividades de reforzamiento y resolución de consultas. Esta metodología ha demostrado diversas ventajas en las instituciones educativas donde se implementó incrementando el número de estudiantes aprobados por asignatura, mejorando la satisfacción y motivación de los estudiantes, y mostrando ser una herramienta óptima para los estudiantes más capaces (Aguilera, et al., 2017).

\section{Métodos y características de la educación virtual}

La educación a distancia se ha caracterizado desde sus inicios por intentar resolver la separación física entre dicentes y profesores, el primer medio fue el correo, posteriormente se incluyó la radio, la televisión y las grabaciones en vídeo, ahora utilizando la computadora y el desarrollo de las redes para su interconexión (Lima \& Fernández, 2016).

Es indudable que la educación universitaria a nivel mundial ha experimentado un cambio estructural y más aún en el proceso de enseñanza-aprendizaje. En estas condiciones la comunicación relevante es síncrona y asíncrona, en el primer caso, el docente es el emisor y los receptores son los estudiantes y que interactúan de manera simultánea, a través de una plataforma de videoconferencia, que permite la interactividad en el mismo marco temporal, a través del envío de mensajes, vídeo, imágenes y texto. Por otro lado, en la comunicación asíncrona, no hay interacción simultánea entre el emisor y los receptores. Se hace uso de una plataforma virtual (servidor en la nube) donde se almacenan los materiales educativos digitales, para que 
el acceso y comunicación se realice en forma diferida. Además, la experiencia nos indica, que en la comunicación asíncrona se observan nuevas formas de desarrollar el proceso de enseñanza aprendizaje, a través de WhatsApp, Facebook, páginas web, y correos electrónicos.

Sterzer (2020), menciona:

En la realidad actual, el 60\% de la población del mundo tiene acceso online. En algunos países las clases virtuales se pueden desarrollar y observar en tablets que cada alumno posee, en ciertos países las lecciones y tareas se remitirán por email o WhatsApp. Cuanto menos acceso a las tecnologías tengan las familias, más rezagados quedarán los alumnos en el proceso de aprendizaje... En Corea del Sur, la mayoría de las universidades adoptaron la enseñanza online, como el uso de PowerPoint de manera auditiva, presentaciones en tiempo real por medio de WebEx, Zoom, transmisión en vivo a través de YouTube o subir videos en esta última plataforma. En Hong Kong, la implementación de la enseñanza online en la educación superior no es un fenómeno nuevo. Las universidades crearon videos y guías de enseñanza y condujeron workshops online para equipar a la comunidad educativa en la utilización de varias plataformas como Zoom, Skype, Moodle y Google Drive. (p.66-69).

La educación virtual se caracteriza por ser flexible y de fácil uso. Permite el acceso a grandes volúmenes de información y recursos digitales, en cualquier momento y desde cualquier lugar, sin horarios, esto gracias al avance de la ciencia y telecomunicaciones. Refuerza el sentido de colaboración entre estudiantes. Se desarrolla la personalización y seguimiento de aprendizaje de los estudiantes y se experimenta nuevos escenarios de aprendizaje y mayor cobertura. Por otro lado, le demanda más tiempo al profesor, para la realización de actividades síncronas y asíncronas, se requiere una planificación de la clase al mínimo detalle, el profesor universitario debe expresar competencias digitales, para el uso de herramientas Tics de enseñanza - aprendizaje y diversas plataformas virtuales y para ello es necesario el uso de computadora con conexión a internet.

\section{Plataformas de aprendizaje y videoconferencias}

En el mundo moderno las tecnologías de aprendizaje en línea se han convertido en herramientas virtuales importantes para el desarrollo de las experiencias de aprendizajes de los estudiantes en la universidad. En consecuencia, el docente universitario tiene que usar metodologías completamente en línea, alineadas con el proceso de aprendizaje de los estudiantes.

En la siguiente tabla se pueden apreciarlas diversas plataformas de gestión del aprendizaje utilizadas por los docentes universitarios en el proceso de enseñanza-aprendizaje.

Tabla 1

Plataforma de Gestión de Aprendizaje y Videoconferencia

\begin{tabular}{|l|l|}
\hline \multicolumn{1}{|c|}{ Learning Management System } & \multicolumn{1}{|c|}{ Plataformas de videoconferencia } \\
\hline - Canvas & \\
- Google Classrrom & - Webwx \\
- Moodle & - Zoom \\
- Chamilo & - Google Meet \\
- Blackboard & - Microsoft Teams \\
- Moodlerooms & - BigBlueBoton \\
- Office 365 & - Jitsi \\
\hline
\end{tabular}

Fuente: Bartra (2020). PUCP 
Es necesario mencionar que muchas universidades del mundo son usuarios de Moodle y que, según información de Bartra (2020), Moodle como gestor de aprendizaje tiene más de 68 millones de usuarios en el mundo, es la plataforma de gestión de aprendizaje más conocida y popular para el desarrollo de un entorno de aprendizaje formal en la nube. De igual manera menciona que, Canvas es una plataforma líder de LMS y que más de 800 universidades y colegios lo usan en el mundo, Google Classrrom es usado por un número indeterminado de personas, teniendo en cuenta que está disponible gratis para todos los millones de usuarios de Google en el mundo, y que los docentes y los estudiantes, viene usándolo de forma complementaria en el proceso de enseñanza-aprendizaje, y que actualmente es una plataforma intuitiva y amigable. Una clase virtual que integra las TIC para el aprovechamiento pedagógico de las plataformas y herramientas digitales en línea y desarrollar el pensamiento crítico, la creatividad, la innovación, el trabajo colaborativo, resolución de problemas en los estudiantes "puede considerarse un formato de maestro virtual" (Ocaña, 2020. p. 8).

\section{Experiencias de metodologías no presenciales}

Una experiencia importante de la adaptación a las clases no presenciales, se presenta en las universidades de España, en especial en las universidades públicas de Castilla y León, donde se observa recomendaciones de alto nivel sobre la forma de afrontar la planificación de evaluación online desde la aplicación del Moodle por su implantación mayoritaria en el sistema universitario a nivel mundial y especialmente en España, entre ellos se trabaja con diversas herramientas de evaluación online como las tareas, cuestionarios, foro, taller , lección en Moodle, cuestionarios externos al campus virtual realizados con herramientas ofimáticas insti-tucionales (como Google Suite o Microsoft Office 365) y video conferencias en general (García, Correll, Avella, y Grande, 2020).

En la Universidad Nacional del Centro del Perú puede verificarse su experiencia respecto a la metodología no presencial, a través de lo que señala el informe de monitoreo de las clases virtuales que presenta el Dr. Huamán (2020), Coordinador Académico de la Facultad de Educación al director de la Oficina de gestión e innovación académica, en donde menciona lo siguiente: Los docentes de las siete carreras profesionales muestran un desempeño sobresaliente, y han cumplido con:

1) Organizar los materiales de enseñanza por semanas y temáticas en concordancia al sílabo en la plataforma Moodle y en Teams y cumplen al 100\% con el avance silábico,

2) Desarrollan las clases sincrónicas a través del MS Teams, en gran mayoría están grabadas y compartidos las clases-video en la plataforma de aprendizaje virtual - Moodle,

3) Usan por lo menos tres recursos virtuales en la plataforma de aprendizaje virtual, como archivos PDF, tareas, lecturas, PPT, videos-clase, foros, etc.

4) Usan diversas formas de participación de los estudiantes, como tareas y foros,

5) Una de las debilidades aún sigue en la parte de evaluación, los docentes han empleado diversas formas y recursos de evaluación y su registro respectivo. Todos los docentes han utilizado el WhatsApp como recurso alternativo, para coordinar, enviar y recibir trabajos por los problemas de conectividad y acceso a internet por parte de los estudiantes, de conformidad a los lineamientos académicos de la Facultad. El MS Teams es el programa de mayor uso por los docentes y estudiantes, para la enseñanza aprendizaje y evaluación, en 
tanto que la plataforma Moodle aún tiene dificultades de acceso y su uso por problemas de conectividad.

Otras de las experiencias observadas fue el caso de la Escuela de Estudios Generales de la Universidad Mayor de San Marcos en donde, al amparo de la Resolución del Consejo Directivo No 039-2020-SUNEDU-CD y de la Resolución Viceministerial No 085-2020-MINEDU se adecuaron todos los cursos y programas a la modalidad no presencial. Así, tal como señala la vicerrectora académica de pregrado Dra. Elizabeth Canales en su Informe de Gestión Académica durante la clausura del año académico 2020, se adecuaron los planes de estudio de las 5 áreas que conforman esta escuela, indicando además que ha sido una de las fortalezas de la universidad "la creatividad e innovación del docente y estudiante sanmarquino para enfrentar nuevos desafíos y escenarios adversos".

En la Escuela de Estudios Generales, se ha empleado el Google Classroom como aula virtual en donde cada docente subía el material de clase y material de trabajo como lecturas, diapositivas, videos; además de asignar, recibir y calificar las tareas (tales como trabajos escritos, foros, evaluaciones de cuestionarios). Es decir, mediante esta plataforma se trabajaron todos los momentos asincrónicos. Mientras que para las clases sincrónicas se empleó el Google Meet fomentándose el trabajo colaborativo y participativo de los estudiantes en cada sesión. En ambas plataformas los estudiantes pueden acceder únicamente mediante su correo institucional.

En el mismo informe Canales (2021) señala que entre las principales dificultades observadas durante el desarrollo del año académico 2020 se han encontrado deficiencias en la conectividad de docentes y estudiantes para el desarrollo académico el cual se debe a la falta de una adecuada banda ancha que no ha sido proporcionado por el Estado al sistema educativo público peruano.

También se observó la experiencia curricular "Construcción y validación de instrumentos de evaluación educativa" del Programa semipresencial de maestría de Docencia Universitaria - Lima Norte - de la Escuela de Posgrado de la Universidad César Vallejo, el diseño metodológico e instruccional responde a la modalidad semipresencial, necesidades y expectativas de los estudiantes, se integran herramientas y recursos digitales para el proceso de enseñanza-aprendizaje, y cuenta con la plataforma digital de aprendizaje "Clementina" diseñada bajo el modelo Flipped Classroom también conocido como metodología del aula invertida", que es el aula virtual para el aprendizaje asíncrono. Aquí se alojan los recursos digitales de aprendizaje, como presentaciones, documentos, videos, tutoriales, lecturas obligatorias, opcionales y enlaces de interés en formato PDF, y se fomenta el aprendizaje social, colaborativo y trabajo en equipo. Los estudiantes acceden con el usuario y contraseña generado por el superadministrador de la plataforma y pueden acceder desde cualquier dispositivo como, laptop, PC, tableta o celular. Para la gestión administrativa, financiera y matricula, los estudiantes cuentan con la plataforma TRILCE.

El docente-tutor aplica estrategias con el uso de las TIC, que promueven el autoaprendizaje, la autonomía y autorregulación en el proceso de aprendizaje y el desarrollo de la competencia a través de los foros de análisis y la presentación de un producto integrador individual y grupal. Además, brinda la posibilidad de acceso e interacción con redes de conocimiento a través de Internet, estimulando el desarrollo de habilidades, destrezas, capacidades y competencias de las experiencias curriculares y digitales; las clases son grabadas y están disponibles durante todo el semestre para su revisión y reforzamiento. 
En esta modalidad, en cada semestre académico durante un fin de semana, a los participantes se les programa sesiones presenciales en uno de los campus de la universidad. De esta manera se complementan las horas virtuales programadas y los estudiantes y docentes asisten al aula física, de acuerdo al plan de estudios de cada programa, como se indica en el prospecto de admisión 2020-II de los programas de maestría y doctorado en la página web de la Universidad César Vallejo. En este contexto de la pandemia, los talleres presenciales fueron reemplazados por videoconferencias realizadas con la herramienta Zoom una vez por semana. Durante la primera semana se realizaban dos talleres de reforzamiento académico extracurricular. Esta experiencia se puede verificar en el informe del período académico 2020 II, presentado por el Coordinador de maestrías de la Escuela de Posgrado de la Universidad César Vallejo Dr. Morales.

Del mismo modo, en la Universidad Privada del Norte se observó que durante el semestre académico 2020-2 se estableció la metodología del aula invertida para el desarrollo de las clases remotas, a través de lineamientos informados por cada coordinador de carrera en donde mencionan que la utilización de material asincrónico, debe ser gestionada por cada profesor de un curso asignado, subiendo el contenido a la plataforma virtual utilizada por la institución: Blackboard Collaborate, elementos como el diseño de la sesión de aprendizaje, las diapositivas del tema a tratar en formato pdf, una lectura o un podscat referente al tema de estudio y la grabación de video sobre el desarrollo de los PPTs elaborados para la sesión de aprendizaje, con una anticipación de 7 días ante de la fecha de clase, y con el monitoreo y reporte del área de auditoria. Respecto al momento sincrónico de la clase, cada profesor debe asistir en el horario programado para el curso, desarrollando actividades que refuercen el tema de estudio y principalmente absolviendo todas las consultas que los estudiantes tengan preparadas luego del análisis de los materiales trabajados de forma asincrónica los que deben haber sido revisados con anterioridad al inicio de la sesión. La implementación de la metodología del aula invertida en la Universidad Privada del Norte durante el semestre 2020-2 brindó mayor flexibilidad a los estudiantes que tuvieron que buscar oportunidades laborares para solventar el pago de las cuotas de la universidad presionados por la coyuntura establecida por la pandemia Covid-19, y a su vez los motivó a un rol más activo y colaborativo con sus pares, propiciando y elevando el aprendizaje autónomo entre los estudiantes. Todos estos puntos fueron manifestados por la mayoría de los profesores de la universidad en la última reunión docente del ciclo 2020-2.

\section{Discusión}

En los procesos de virtualización de los cursos virtuales para el proceso de enseñanza-aprendizaje en las universidades y el proceso de adaptación de las mismas, se generaron desencuentros entre el docente y el estudiante donde se experimentó una interacción pasiva, que puede ser explicado por problemas eminentemente tecnológicos, acceso a internet, la falta de habilidades, destrezas y capacidades en competencias digitales y finalmente factores socio afectivas.

En consecuencia, en plena pandemia muchas universidades del país y del mundo han venido adaptándose a una situación de crisis en la educación virtual, que garantizaba mínimamente cierto éxito en los procesos de aprendizaje y mostrándose de alguna manera empáticos con la realidad socioemocional de los estudiantes universitarios. Al respecto Pardo y Cobo (2020, p. 8) citados por Baladrón, Correyero y Manchado (2020, p. 271) mencionan que "muchas universidades han improvisado, llegando tarde o con una preparación insuficiente a esta 
adaptación, aplicando voluntarismo y replicando en la virtualidad modelos de aprendizaje que ya están siendo obsoletos en la presencialidad".

Existen investigadores que han presentado datos que indican que más del $85 \%$ de los educadores que imparten cursos en línea sienten que los estudiantes aprenden tanto como lo harían en las aulas. El error más grande, dicen los expertos, es tratar de hacer que el aprendizaje en línea sea lo mismo que el aprendizaje en el aula, cuando en realidad deberían ser muy diferentes (Tompkins, 2020, citado por Abreu, 2020, p. 13).

Si bien fue importante el uso de diversos recursos didácticos digitales en la educación a distancia y virtual, no debemos dejar de mencionar que en las universidades especialmente las públicas se observó inequidad a la hora de la implementación y hacer uso de las tecnologías de la información y comunicación y el acceso al internet, por parte del estado peruano. Además, la educación a distancia no aseguraba calidad educativa y el logro de los aprendizajes en los estudiantes, tal como se desarrolla en una educación presencial.

Además, Faulder (2011) y Jimoyaiannis (2010) citados por López, Couso y Simarro (s/f) manifiestan que

algunas investigaciones han señalado claramente que el uso de tecnologías digitales no implica de forma automática una mejora de los procesos de enseñanza, ya que la manera en que se usan no depende solamente de la herramienta en sí, sino de las creencias y los modelos didácticos del profesorado que las adopta.

Es necesario que el docente universitario en las circunstancias actuales reflexione y realice un análisis crítico sobre su práctica metodológica en el proceso de enseñanza-aprendizaje, si en esta realidad de la pandemia, la experiencia profesional de trabajo virtual fue ineficaz, aburrida, de limitada interacción con el estudiante y centrada esencialmente en el docente por lo que los estudiantes recibieron la información en forma pasiva, tal como indica Ocaña (2020) "los diversos trabajos de investigación y la literatura académica demuestran los múltiples beneficios pedagógicos de un adecuado diseño de aprendizajes que incorporan la tecnología generando eficiencia en el aprendizaje" (p. 8).

En relación a los casos observados en las universidades españolas en el contexto de la pandemia por la COVID-19, Baladrón, Correyero y Manchado (2020) mencionan que

... durante el confinamiento, el 30\% del profesorado universitario español impartió docencia no presencial todos los días (el $55 \%$ varias veces a la semana) cuando el $60 \%$ de docentes nunca había impartido clases online (Ibercampus, 2020). Este estudio también constata que al $10 \%$ de los docentes le ha costado trabajo enseñar a distancia, por no contar con conocimientos suficientes sobre enseñanza online, o que el $72 \%$ del profesorado ha adquirido conocimientos con cursos de la propia universidad (el 56,5\% también con ayuda de compañeros a través de redes). Todos los docentes consultados afirman que, en general, sus conocimientos del entorno docente online se han incrementado en más de un 50\% respecto a lo que sabían antes y el 32\% se considera suficientemente preparado para seguir impartiendo docencia no presencial tras este período. Estos datos pondrían de manifiesto que, en general, los profesores se han adaptado bien y rápido a las nuevas metodologías y que se han familiarizado sin problemas con herramientas como Moodle, Blackboard Collaborate o Teams, que han sido las más utilizadas (p. 271). 
Al margen de la implementación tecnológica para el desarrollo de la educación virtual desarrollado en las universidades, es necesario que en esta situación de emergencia global se desarrollen procesos de cambio en las formas de enseñanza remota y naturalizar la cultura digital en la educación superior. Esta ocasión debe ser pensada a la vez como un reto institucional, uno para los docentes y también un reto de los propios alumnos. (Pardo y Cobo, 2020, p. 13)

\section{Conclusiones}

La crisis sanitaria mundial ha generado importantes cambios cualitativos en la actividad pedagógica y la metodología del docente universitario principalmente por la mediación tecnológica a través de las estrategias asíncronas (foros, blog, comunidades virtuales, etc.) y síncronas basados en el aprendizaje social, modelo didáctico interactivo y colaborativo asistido por computadora, laptop, Tablet, teléfonos inteligentes, u otros medios tecnológicos, desarrollando sus clases desde plataformas de gestión de aprendizajes (Canvas, Google Classrrom, Moodle, Chamilo, Blackboard, etc.) y videoconferencias (Webex, Zoom, Google Meet, Microsoft Teams, etc.). Todo esto conllevó al profesor universitario a entender los problemas de conectividad dentro de la crisis de la educación a distancia y virtual. Estas estrategias de interacción virtual a través de las tecnologías de la información y comunicación o herramientas digitales para el aprendizaje, aseguraron la continuidad de la educación en tiempos del COVID-19.

La pandemia producida por el COVID-19, ha evidenciado la brecha digital entre estudiantes y docentes, en este contexto, Eche (2017) "recomienda la capacitación docente continúa en el uso adecuado de herramientas TIC, Google Apps para educación, nuevos enfoques y metodologías de aprendizaje, aulas virtuales de aprendizaje, aplicaciones y uso adecuado de PC, Tablet, teléfonos inteligentes para su uso en la práctica pedagógica” (p. 99), por lo que se hace urgente y necesario que las instituciones universitarias realicen acciones formativas sostenidas que integren enfoques y estrategias metodológicas con las tecnologías de la información y comunicación en el proceso de enseñanza-aprendizaje, a partir de la identificación de las fortalezas y debilidades en los docentes.

Para la continuidad del servicio educativo universitario a través de la educación a distancia y virtual, requiere necesariamente que el docente universitario esté en la capacidad de diseñar entornos virtuales de aprendizaje multimedia (creación de actividades multimedia, aula virtual, etc.), uso de herramientas TIC en educación (diseñar recursos educativos digitales para la creación de mapas mentales, diagramas, presentaciones, etc.), producir material digital interactivo para el aprendizaje virtual (libros interactivos, multimedia, LIM, eXelearning, EducaPlay, Cuadernia, etc.) y aplicaciones web (Powtoon, Google Drive y Suite, Genially, formularios interactvos, etc.), y desarrolle estrategias docentes con el uso pedagógico adecuado de estas herramientas que garanticen durante el proceso de enseñanza-aprendizaje el logro de aprendizajes y desarrollo de competencias en beneficio de los estudiantes en el contexto actual. 
Abreu, J. L. (2020). Tiempos de Coronavirus: La Educación en Línea como Respuesta a la Crisis. International Journal of Good Conscience. (1) 1-15. http://www.spentamexico.org/v15-n1/ A1.15(1)1-15.pdf

Aguilera, C., Manzano A., Martínez, I., Lozano, M. y Casiano, C. (2017). El modelo flipped classroom. International Journal of Developmental and Educational Psychology, 4(1), 261-266. https://www.redalyc.org/pdf/3498/349853537027.pdf

Area-Moreira, M. (2019). La enseñanza universitaria digital: fundamentos pedagógicos y tendencias actuales.

https://riull.ull.es/xmlui/bitstream/handle/915/13247/La\%2oense\%C3\%B1anza\%20 universitaria\%20digital\%20-\%20Manuel\%20Area-Moreira\%20\%28marzo\%202019\%29. pdf? sequence $=1 \&$ isAllowed $=y$

Baladrón, A. J., Correyero, B. y Manchado, B. (2020). La transformación digital de la docencia universitaria en comunicación durante la crisis de la COVID-19 en España: una aproximación desde la perspectiva del alumnado. Revista Latina de Comunicación Social, 78, 265-287. http:// nuevaepoca.revistalatinacs.org/index.php/revista/article/view/294

Bartra, G. (2020). Prácticas docentes, modalidad no presencial. PUCP, Ingeniería de telecomunicaciones.

Canales, E. (2021, 10 de febrero). Avances y desafíos. Informe de Gestión Académica. [Informe] Ceremonia de clausura del año académico 2020 UNMSM. https://viceacademico.unmsm. edu.pe/wp-content/uploads/2021/o2/Bolet\%C3\%ADn-N\%C2\%Bo-6-Clausura-delA\%C3\%B10-Acad\%C3\%Agmico-2020-1.pdf

Copertari, S., y Sgreccia, N. (2011). Postgrados a distancia y virtualización en la Universidad Nacional de Rosario. Educación y humanismo, 13(20), 14-32.

http://revistas.unisimon.edu.co/index.php/educacion/article/view/2279

Eche, P. (2017). Uso del Software "El Mundo Interactivo de Z" y su Influencia en el Aprendizaje de la Matemática en los Estudiantes del Primer Grado de la Institución Educativa N 5049 Emma Dettmann de Gutiérrez del Distrito del Callao. Año 2015 [Tesis de maestría]. Universidad nacional de Educación Enrique Guzmán y Valle

Garcés, J. E. \& Jair Duque, E. J. (2007). Metodología para el análisis y la revisión crítica de artículos de investigación. Innovar, 17(29) 184-194

García, F. J., Correll, A., Avella, V. y Grande, M. (2020). La evaluación online en la educación superior en tiempos de la COVID-19. Ediciones Universidad de Salamanca. 21 (12) 1-26

https://revistas.usal.es/index.php/eks/article/view/eks20202112

Huamán, L. (2020). Resultados del segundo Monitoreo de la labor docente en clases virtuales de la Facultad de Educación. Oficio No O41-DDE-FE-UNCP, 24 de agosto.

Lima, S., \& Fernández, F. (2016). La Educación a Distancia en Entornos Virtuales de Enseñanza Aprendizaje. Reflexiones Didácticas. Tecnología Educativa, 1(1), 43-54. http://tecedu.uho. edu.cu/

López, V., Couso, D. y Simarro, C. (s/f). Educación STEM en y para el mundo digital. Cómo y por qué llevar las herramientas digitales a las aulas de ciencias, matemáticas y tecnologías. Revista de Educación a distancia

https://www.um.es/ead/red/58/lopez_et_al.pdf

Ocaña-Fernández, Y., Valenzuela-Fernández, L., \& Morillo-Flores, J. (2020). La competencia digital en el docente universitario. Propósitos y Representaciones, 8(1), 455: Revista de psicología educativa | Journal of Educational Psychology ISSN 2310-4635 Universidad San Ignacio de Loyola. DOI: http://dx.doi.org/10.20511/pyr2020.v8n1.455 
Pardo H. y Cobo, C. (2020). Expandir la universidad más allá de la enseñanza remota de emergencia. Ideas hacia un modelo híbrido post-pandemia. Outliers School

https://outliersschool.net/wp-content/uploads/2020/05/Expandir_la_universidad.pdf

Peña, T. y Pirela, J. (2007). La complejidad del análisis documental. Información, cultura y sociedad: revista del Instituto de Investigaciones Bibliotecológicas. (16) 55-81. Disponible en https:// www.redalyc.org/pdf/2630/263019682004.pdf

Pinheiro, I. M (2020). Estado da arte sobre o ensino de enfermagem e os desafios do uso de tecnologias remotas em época de pandemia do corona vírus. J Hum Growth Dev. 30(1):141147

DOI: http://doi.org/10.7322/jhgd.v30.10087

Schnotz, W. (2002). Towards and integrated view of learning from text and visual displays. Educational Psychology Review. 14 (1) 101-120. file:///Users/oscar/Downloads/Schnotz2002_ TowardsAnIntegratedView.pdf

Skulmowski, A. y Daniel. G. (2020). COVID19 as an accelerator for digitalization at a German university: Establishing hybrid campuses in times of crisis. Hum Behav Q Emerg Tech. 2. (3) 212-216

https://doi.org/10.1002/hbe2.201

Sterzer, S. (2020). Impacto del coronavirus en el sistema educativo: Ejemplos en el continente asiático. Red Sociales, Revista del Departamento de Ciencias Sociales, 7 (2) 64-74.

https://ri.unlu.edu.ar/xmlui/bitstream/handle/rediunlu/751/10.-Impacto-delcoronavirus-en-el-sistema-educativo_-ejemplos-en-el-continente-asi\% $\mathrm{C}_{3} \% \mathrm{~A}_{1}$ tico.. pdf?sequence $=1$ \&isAllowed $=y$

Suárez Urquijo, S. L., Flórez Álvarez, J. y Peláez, A. M. (enero-junio, 2019). Las competencias digitales docentes y su importancia en ambientes virtuales de aprendizaje. Revista Reflexiones y Saberes (10) 33-41.

Varguillas, C., y Bravo, P. (2020). Virtualidad como herramienta de apoyo a la presencialidad: análisis desde la mirada estudiantil. Revista de Ciencias sociales, XXVI (1) 219-232.

http://produccioncientificaluz.org/index.php/rcs/article/view/31321/32371

Vásquez, E. (2015). El reto de la formación docente para el uso de dispositivos digitales móviles en la educación superior. Perspectiva Educacional. Formación de profesores 54 (1) 149-162

https://www.redalyc.org/pdf/3333/333333042010.pdf

(C) Los autores. Este artículo es publicado por la Horizonte de la Ciencia de la Unidad de Posgrado de la Facultad de Educación de la Universidad Nacional del Centro del Perú. Este es un artículo de acceso abierto, distribuido bajo los términos de la Licencia Atribución-No Comercial 4.0 Internacional.(https://creativecommons.org/licenses/by-nc/4.o/), que permite el uso no comercial y distribución en cualquier medio, siempre que la obra original sea debidamente citada. 